\title{
Skin Temperature Prediction in Lower Limb Prostheses
}

\author{
Neha Mathur, Student Member, IEEE, Ivan Glesk, Senior Member, IEEE, and Arjan Buis
}

\begin{abstract}
Increased temperature and perspiration within a prosthetic socket is a common complaint of many amputees. The heat dissipation in prosthetic sockets is greatly influenced by the thermal conductive properties of the socket and interface liner materials. These materials influence the body's temperature regulation mechanism and might be the reason for thermal discomfort in prosthetic sockets. Monitoring interface temperature at skin level is notoriously complicated. The problem might be considered notorious because embedding wires and sensors in an elastomer eventually results in elastomer failures because of the high strain induced when donning a liner (amputees roll the liners onto their limbs). Another reason is because placing sensors and wires directly against the skin could cause irritation and chaffing over just a short period of time. We describe a route wherein if the thermal properties of the socket and liner materials are known, the in-socket residual limb temperature could be accurately predicted by monitoring the temperature between socket and liner rather than skin and liner using the Gaussian process technique.
\end{abstract}

Index Terms-Gaussian process for machine learning (GPML), lower limb prosthetics, modeling, temperature.

\section{INTRODUCTION}

$\mathbf{M}$ ONITORING and predicting the residual limb skin health in lower limb amputees is of principal importance as the socket of the prosthesis creates a warm and humid microenvironment that encourages growth of bacteria and skin breakdown [1], [2]. Elevated residual limb skin temperature is considered one of the major factors that could affect the health of soft tissues in that region [3]-[7]. Studies on temperature within the prosthetic sockets of transtibial prosthetic users have been described by Peery et al. [8], [9]. They investigated the in-socket temperature of five transtibial amputees at 14 different locations on the residual limb and at four different stages, i.e., donning, steady-state resting, initial walking, and steadystate walking. Their results indicated that the thermal dissipation characteristics of socket and liner restrict heat loss from the residual limb and the temperature increase is larger in areas where there is more muscle bulk. It was also seen that different socket and liner materials affect the temperature increase

Manuscript received May 15, 2014; revised September 24, 2014; accepted November 2, 2014. Date of publication November 7, 2014; date of current version December 31,2015. This work was supported by the Engineering and Physical Sciences Research Council under the Doctoral Training Grant and the Centre for Excellence in Rehabilitation Research. Also, support for the climate chamber was given by the University of Glasgow.

N. Mathur and I. Glesk are with the Department of Electronic and Electrical Engineering, University of Strathclyde, Glasgow G11XW, U.K. (e-mail: neha.mathur@strath.ac.uk; ivan.glesk@strath.ac.uk).

A. Buis is with the Department of Biomedical Engineering, University of Strathclyde, Glasgow G40NW, U.K. (e-mail: arjan.buis@strath.ac.uk).

Digital Object Identifier 10.1109/JBHI.2014.2368774 in the residual limb differently. Also these temperature rises were different between patients. The impact of environmental factors has also been investigated by Klute et al. [10], [11] by looking at the in-socket residual limb temperature at four locations throughout a whole day. Simultaneously, they recorded environmental temperature, humidity, and also the activity of the patient. It was found that in-socket residual limb temperature increased gradually throughout the day, and an increase in activity caused a further increase in temperature. Also, the environmental humidity and temperature influence the perception of whole body and residual limb thermal comfort [12]. Limitations to this study were small sample size and the variation of prosthetic liners and socket materials between patients. The population on which these studies were done was small. Hence, a generalized statement about the temperature of residual limb skin cannot be made.

Limited research has been done on the thermal behavior of the socket and liner materials and their effect on stump skin temperatures [13]-[16]. Klute et al. [17] investigated the thermal conductivity of different liner and socket materials. Their investigations disclosed that there was a large variation in the thermal conductivity of liner materials, whereas the prosthetic socket materials-thermoplastic and carbon fiber-had similar thermal conductivities. They concluded that liner material selection has a considerable effect on the residual limb skin temperature as compared to the thermoplastic and carbon fiber socket materials which has little effect. The results presented in [17] suggested that some prosthetic components can act as a barrier to conductive heat transfer due to low thermal conductivity. This implies that different liner and socket materials produce a different thermal environment and, hence, can lead to different residual limb skin temperatures [18], [19].

This leads to a hypothesis that if the thermal properties of the socket and liner materials are known, then the in-situ skin temperature could be predicted by monitoring between socket and liner. The purpose of this is to assess whether or not a temperature measurement device can accurately measure the temperature of the residual limb when it is placed either on the inner or outer surface of a prosthetic socket. If that is achievable, then the monitoring of the residual limb skin temperature can be done without undesirable contact of any temperature sensor with the skin thus avoiding any increased skin irritation.

Our approach was to determine an accurate mathematical model using the Gaussian processes for machine learning (GPML) to predict the residual limb skin temperature of the amputee. The temperature measurement device when placed directly in contact with the skin would give the most accurate reading. However, this could create practicality issues with 


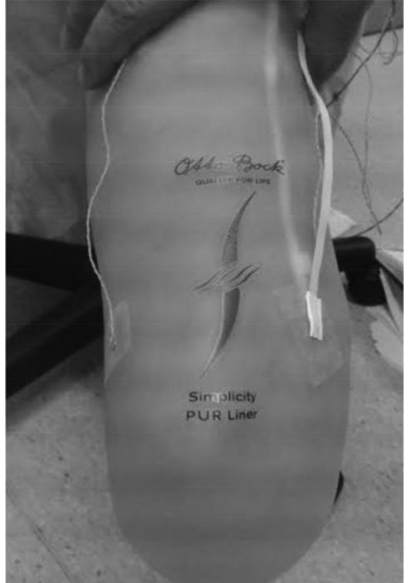

Fig. 1. Placement of the thermocouples on the lateral and medial side of residual limb skin and its corresponding positions on the liner of the transtibial amputee.

normal prosthetic use in a domestic situation such as protruding lead wiring, consistent positioning of sensors, and possible skin irritation. Embedding sensors and wires in the hard prosthetic socket is possible during the manufacturing process for prosthetic sockets. This would eliminate the practicality issues as described earlier. In addition, no damage to the device would occur through donning and doffing and the longevity of the device would not be impaired.

The aims of this project are to investigate how differing activity levels and ambient temperatures influence the in-socket temperature. It would be then investigated that whether the predictive model developed is accurate enough and its response time and repeatability are also sufficient.

\section{METHOD}

To investigate the correlation between the position of thermocouples (skin and in-socket), one transtibial traumatic amputee was recruited to perform in a 35-min laboratory protocol. The investigation was implemented following ethical approval granted by the University of Strathclyde Ethics Committee (Ref UEC13/04).

The subject was a 68-year-old male who weighed $70 \mathrm{~kg}$ and wore a 6-mm Otto Bock HealthCare $\mathrm{GmbH}$ Technogel liner with a resin laminate socket. For the experiment, the subject was dressed in shorts and t-shirt. Hence, it should be noted that there was no extra clothing layer on the prosthesis. To monitor and record the skin and in-socket temperatures, four K-type thermocouples via a data logger (type HH1384; Omega Engineering) were used. Two thermocouples were taped onto the residual limb in lateral and medial position. The other two thermocouples were put on the corresponding positions on the liner (in-socket). This is indicated in Fig. 1. Data from the four channels were simultaneously collected at $0.5 \mathrm{~Hz}$ at a predetermined ambient temperature.

After the thermocouple heads were secured with tape, the prosthesis was donned with the thermocouple wires exiting the proximal edge of the socket. The subject was asked to complete

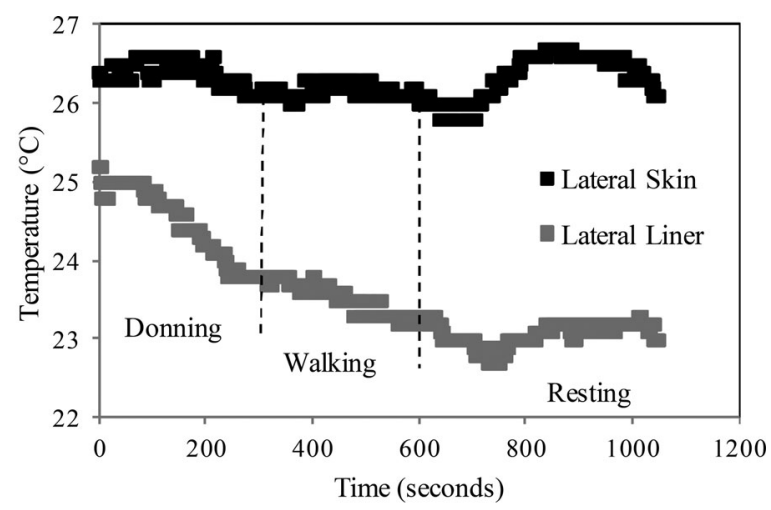

(a)

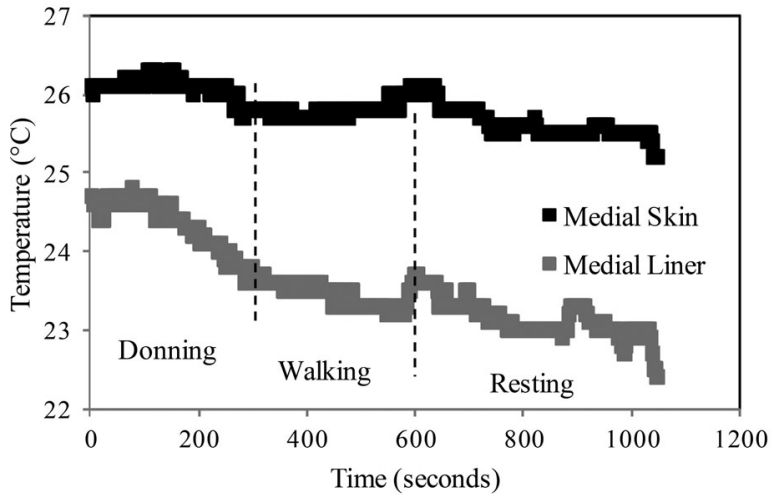

(b)

Fig. 2. Profiles of residual limb skin and liner temperature at ambient temperature $10^{\circ} \mathrm{C}$ on (a) lateral side and (b) medial side.

the following protocol: resting (sitting) for $10 \mathrm{~min}$, walk at selfselected pace of $0.62 \mathrm{~m} / \mathrm{s}$ on a treadmill for $10 \mathrm{~min}$, and finally rest for $15 \mathrm{~min}$. The residual limb skin and the socket temperatures were sampled at $0.5 \mathrm{~Hz}$ for the entire 35-min protocol. For analysis purposes, three steady-state periods were defined as the last minute of each period: initial rest, walking, and final rest. The temperature profile of the residual limb skin and liner was analyzed at different ambient temperatures to see how closely they are correlated. This study was conducted in Scotland for the Spring/Summer profile where the ambient temperature ranges from approximately $10{ }^{\circ} \mathrm{C}$ to $25^{\circ} \mathrm{C}$. Hence, the temperatures from this range were picked. We conducted the experiment at $10{ }^{\circ} \mathrm{C}$ and then repeated for $15^{\circ} \mathrm{C}, 20^{\circ} \mathrm{C}$, and $25^{\circ} \mathrm{C}$ (Dataset A). The experiments were conducted again after a time span of two months to confirm the influence of ambient temperature on the residual limb skin temperature (Dataset B). All experiments were conducted in a climate-controlled chamber with zero wind velocity and $40 \%$ humidity level.

\section{RAW DATA}

The temperature profiles of the liner and residual limb skin at ambient temperatures of $10^{\circ} \mathrm{C}, 15^{\circ} \mathrm{C}, 20^{\circ} \mathrm{C}$, and $25^{\circ} \mathrm{C}$ from set B are shown in Figs. 2-5.

From the studies on the amputee subject, it is seen that donning causes a moderate temperature increase as also reported in [20], walking causes a significant increase, and the rest periods following activities must be substantially long to return the limb 


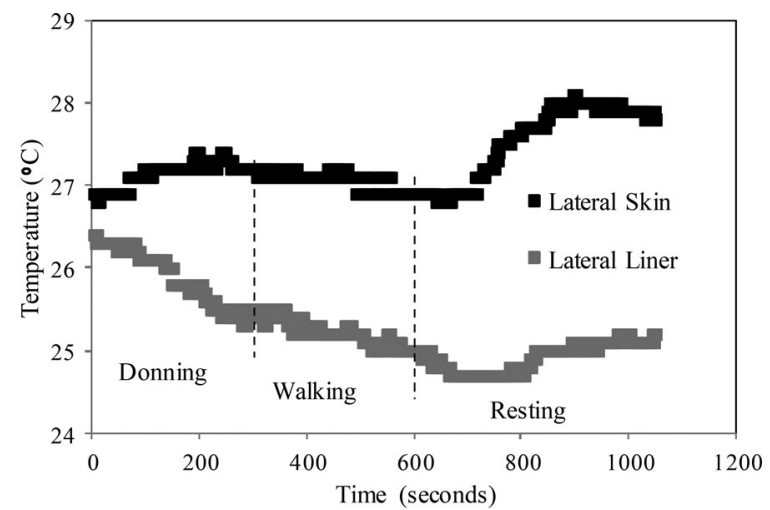

(a)

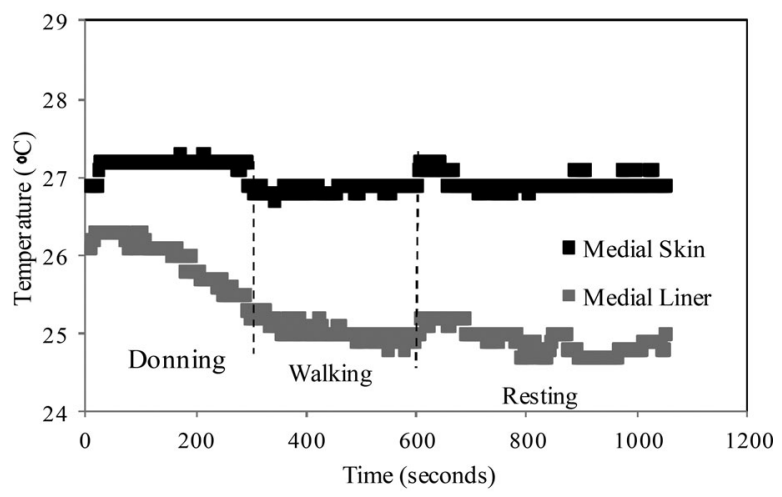

(b)

Fig. 3. Profiles of residual limb skin and liner temperature at ambient temperature $15{ }^{\circ} \mathrm{C}$ on (a) lateral side and (b) medial side.

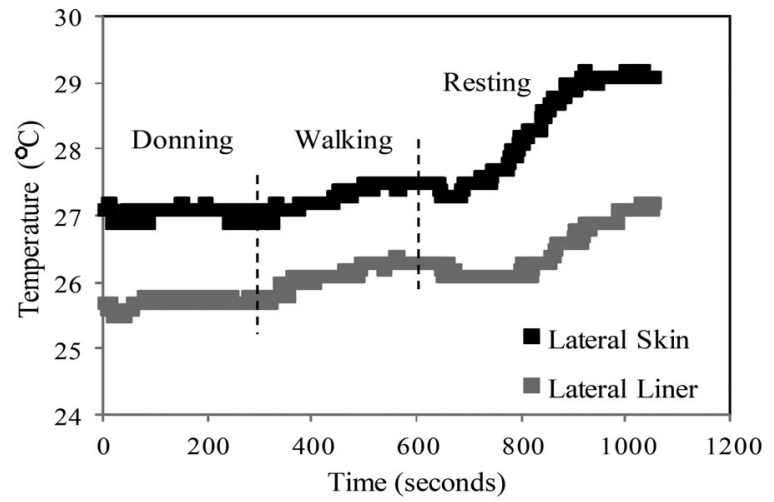

(a)

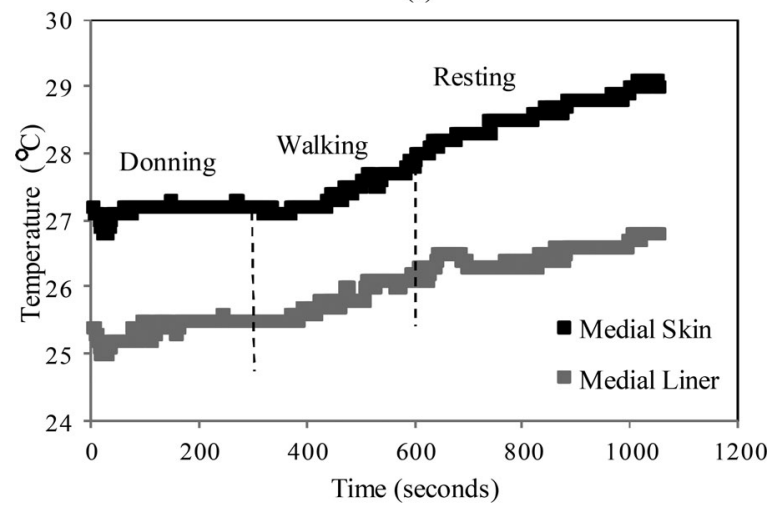

(b)

Fig. 4. Profiles of residual limb skin and liner temperature at ambient temperature $20^{\circ} \mathrm{C}$ on (a) lateral side and (b) medial side.

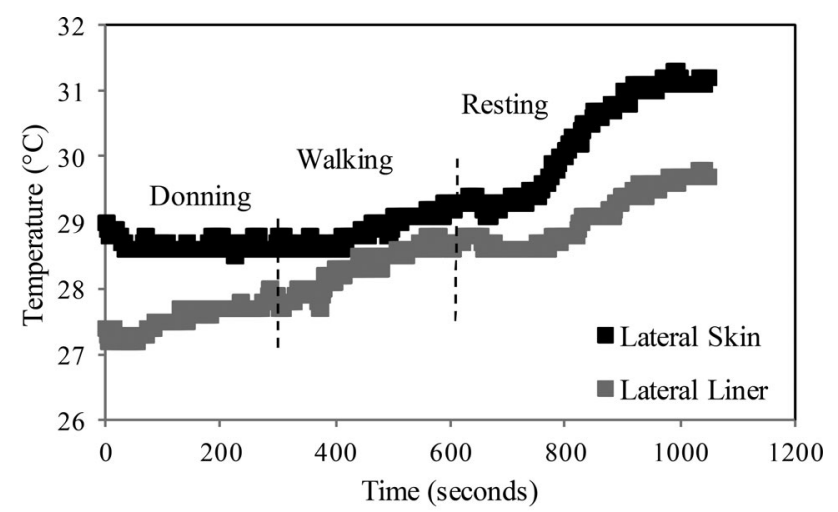

(a)

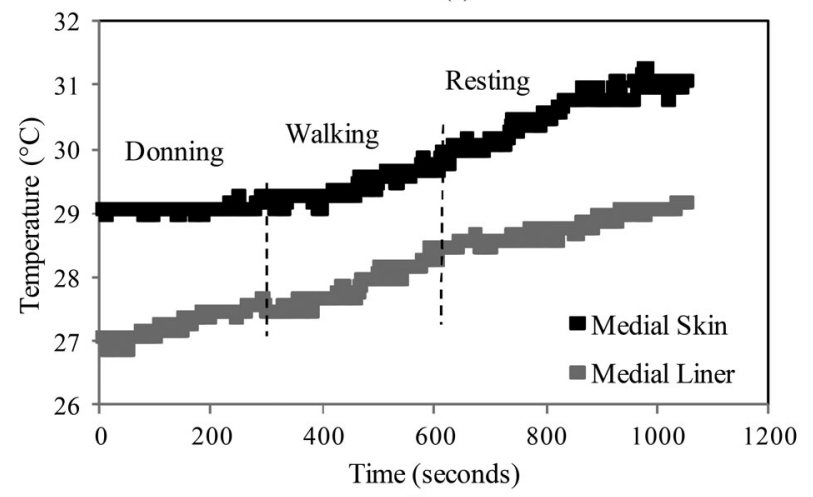

(b)

Fig. 5. Profiles of residual limb skin and liner temperature at ambient temperature $25^{\circ} \mathrm{C}$ on (a) lateral side and (b) medial side.

to temperatures before donning the prosthesis. Figs. 2 and 3 indicate that the residual limb temperature profile for ambient temperatures of $10{ }^{\circ} \mathrm{C}$ and $15^{\circ} \mathrm{C}$ has a similar pattern of being steady throughout the experiment. However, this temperature profile of the residual limb is significantly different from that at ambient temperatures of $20{ }^{\circ} \mathrm{C}$ and $25^{\circ} \mathrm{C}$ as indicated in Figs. 4 and 5.

Both the lateral and medial residual limb skin temperatures showed a steady increase in the temperature throughout the experiment. After the end of the experiment, the temperatures in both lateral and medial side were $2.1{ }^{\circ} \mathrm{C}$ higher than the starting.

It can be seen from these studies on amputee subject that the residual limb skin temperature behavior is a function of ambient temperature. This reflects that though the human body self regulates to maintain a stable internal environment despite changes in the external environment, in case of prosthetic users there are layers of liner and socket materials which inhibit the body's ability to thermoregulate effectively.

Since, the temperature profiles of the residual limb are almost similar for the ambient temperature pairs of $10{ }^{\circ} \mathrm{C}, 15^{\circ} \mathrm{C}$ and $20^{\circ} \mathrm{C}, 25^{\circ} \mathrm{C}$, the Gaussian predictive model at ambient temperatures of $10^{\circ} \mathrm{C}$ and $25^{\circ} \mathrm{C}$ are only discussed in this study.

\section{Gaussian Process FOR Machine LEARNING}

The data from the data logger indicated that at any given ambient temperature, the trace of the liner temperature follows 
that of the residual limb skin as in Figs. 2-5. This suggested a possibility to model the liner temperature as a function of skin temperature and create a mathematical model of the same. Processing was performed with custom developed software (using MATLAB, Mathworks). The model designed takes the liner temperature as the input $x$ and the predicted output is the residual limb skin temperature $y$.

The predictive model was developed using the GPML [21]. Similar with other regression methods, the goal of Gaussian process regression is to infer a continuous function $f(x)$ from a training set of input-output pairs in a supervised learning context. The key assumption in Gaussian process modeling is that our data can be represented as a sample from a multivariate Gaussian distribution. A Gaussian process model infers a joint probability distribution over all possible outputs for all inputs. This form enables the implementation of Bayesian framework in a simple way [22], [23]. Bayes' theorem states that the posterior probability of a condition is given by the product of the prior probability and the likelihood in the light of the evidence. This can be written as

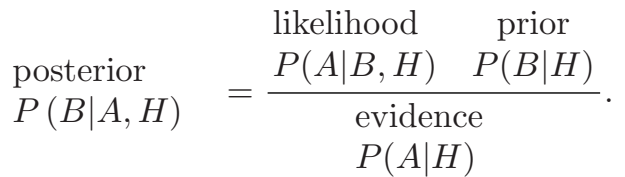

$P(B \mid A, H)$ is the posterior probability that statement $B$ is true, given that condition $A$ is observed and that hypothesis $H$ is correct. $P(A \mid B, H)$ is the probability of observing $A$ if $B$ is true and $H$ is correct, which is called the likelihood. $P(B \mid H)$ is the prior probability of $B$ being true, without having made any observations. $P(A \mid H)$ is the evidence: the probability of observing $A$ if hypothesis $H$ is correct [23].

The inference of a joint probability distribution function involves deducing a number of quantities called the hyperparameters $\Theta$. These hyperparameters are an indication of the precision and relevance of the input parameters for predicting the output. Thus, the aim in a Gaussian process model is to choose model parameters for which the probability of the training data is maximized [22].

If the training data contain $N$ points comprising of their set of targets $y_{N}$ with their corresponding input $x_{N}$, then the Gaussian model is defined by an $N$-dimensional covariance matrix $C_{N}$. The covariance matrix is basically indicative of the closeness to each other outputs for different inputs, taking into account the model parameters. This allows predictions of outputs $y_{*}$ to be made, based on the difference between the new inputs $x_{*}$ and those seen in the training data. Each element of $C_{N}$ is defined by covariance function $C_{f}$, which is a function of inputs and hyperparameters [22]-[24]. For the element $i j$ in covariance matrix, $C_{i j}=C_{f}\left(x_{i}, x_{j}, \Theta\right)$. The covariance function can be user defined. In this study, the covariance function that was used was

$$
C_{f}=\theta_{1} e^{-\frac{\left(x_{i}-x_{j}\right)^{2}}{2 l^{2}}}+\sigma_{n}^{2} \delta_{i j}
$$

where the set of hyperparameters $\Theta=\left\{\theta_{1}, l, \sigma_{n}\right\}$ and $\delta_{i j}$ is a delta function whose value is zero for all $i \neq j$. The first term in the above equation allows the closeness of two outputs to be related to the closeness of the inputs. The length scale $l$ for an input parameter indicates how much the output will vary relative to changes in an input. The initial values of the hyperparameters were selected as $[0.1,1,1]$. To prepare for Gaussian process regression, we calculate the covariance function, (2), for all possible combinations of these points, summarizing in the matrices in the following equations:

$$
C_{N}=\left[\begin{array}{cccc}
C_{f}\left(x_{1}, x_{1}\right) & C_{f}\left(x_{1}, x_{2}\right) & \cdots & C_{f}\left(x_{1}, x_{N}\right) \\
C_{f}\left(x_{2}, x_{1}\right) & C_{f}\left(x_{2}, x_{2}\right) & \cdots & C_{f}\left(x_{2}, x_{N}\right) \\
\vdots & \vdots & & \vdots \\
C_{f}\left(x_{N}, x_{1}\right) & C_{f}\left(x_{N}, x_{2}\right) & \cdots & C_{f}\left(x_{N}, x_{N}\right)
\end{array}\right]
$$

$$
\begin{aligned}
C_{N *} & =\left[\begin{array}{llll}
C_{f}\left(x_{*}, x_{1}\right) & C_{f}\left(x_{*}, x_{2}\right) & \ldots & C_{f}\left(x_{*}, x_{N}\right)
\end{array}\right] \\
C_{N * *} & =C_{f}\left(x_{*}, x_{*}\right)
\end{aligned}
$$

Since the key assumption in Gaussian process modeling is that the data can be represented as a sample from a multivariate Gaussian distribution, we have

$$
\left[\begin{array}{l}
y \\
y_{*}
\end{array}\right] \sim\left(0, \quad\left[\begin{array}{ll}
C_{N} & C_{N *}^{T} \\
C_{N *} & C_{N * *}
\end{array}\right]\right)
$$

where $T$ indicates the matrix transposition. The conditional probability $p\left(y_{*} \mid y\right)$, "given the data, how likely is certain prediction for $y_{*}$," follows a Gaussian distribution as

$$
y_{*} \mid y \sim\left(C_{N *} C_{N}^{-1} y, C_{N * *}-C_{N *} C_{N}^{-1} C_{N *}^{T}\right) .
$$

The reliability of the regression depends upon on covariance function and in turn the hyperparameters. Typically, we would not a priori know the values of the hyperparameters. To get the optimal hyperparameters, (1) becomes

$$
P\left(\Theta \mid y_{N}, x_{N}, C_{f}\right)=\frac{P\left(y_{N} \mid x_{N}, C_{f}, \Theta\right), P(\Theta)}{P\left(y_{N} \mid x_{N}, C_{f}\right)} .
$$

Referring to (8), it is apparent that the evidence is independent of hyperparameters and is constant for a given dataset. To find the optimal hyperparameters, the posterior probability is maximized as the prior maybe noninformative. This corresponds to minimizing the log marginal likelihood (nlml) as in (9). Hence, for a particular training set and covariance function, the Gaussian process would select the best hyperparameters that give the best predictions for training data [23], [24]

$$
\log p(y \mid x, \Theta)=-\frac{1}{2} y^{T} C_{N}^{-1} y-\frac{1}{2} \log \left|C_{N}\right|-\frac{n}{2} \log 2 \pi .
$$

Several multivariate optimization algorithms can be utilized to calculate the hyperparameters, such as Laplace's approximation, Markov Chain Monte Carlo sampling, Kullback-Leibler optimal approximation, or the Variational Bayes approximation. In the present study, kernel hyperparameters were optimized by the exact inference technique. For real-valued outputs, it combines the Gaussian process prior with a Gaussian likelihood and perform an exact posterior inference in closed form. 
TABLE I

SUMMARY OF GPML FOR VARIOUS TESTING AND TRAINING SCENARIOS

\begin{tabular}{lcccccc}
\hline \hline $\begin{array}{l}\text { Training } \\
\text { Set }\end{array}$ & $\begin{array}{c}\text { Test } \\
\text { Set }\end{array}$ & $\begin{array}{c}\text { Training } \\
\text { error } \\
\text { (rms) }\end{array}$ & $\begin{array}{c}\text { Test } \\
\text { error } \\
\text { (rms) }\end{array}$ & $\begin{array}{c}\text { Number of } \\
\text { training } \\
\text { points }\end{array}$ & $\begin{array}{c}\text { Number of } \\
\text { test } \\
\text { points }\end{array}$ & $\begin{array}{c}\text { Normalized } \\
\text { log } \\
\text { likelihood }\end{array}$ \\
\hline A & A & 0.142 & 0.142 & 250 & 100 & 0.786 \\
A & A & 0.0924 & 0.0924 & 500 & 100 & 0.893 \\
A & A & 0.0913 & 0.0913 & 750 & 100 & 0.897 \\
A & A & 0.0910 & 0.0910 & 1050 & 100 & 0.895 \\
A & B & 0.0910 & 0.102 & 1050 & 100 & 0.896 \\
\hline \hline
\end{tabular}

\section{Model Generation And Prediction}

The aim of the model is to predict the residual limb skin temperature from the liner temperature. Hence, the input to the model is the liner temperature and the output of the model would be the residual limb skin temperature. To test the predictive capability of a model, it is trained on one set of data and tested on previously unseen data. It is seen from Section II that the skin temperature is dependent on ambient temperature. Hence, individual Gaussian process models for the lateral and medial side of the residual limb were designed, using the principle as described in the previous section for ambient temperatures of $10{ }^{\circ} \mathrm{C}$ and $25^{\circ} \mathrm{C}$. Consider the ambient temperature $10{ }^{\circ} \mathrm{C}$, first the lateral side model was trained on different scenarios to investigate the optimal training required. Table I presents the different testing and training cases presented to the model. Initially the model was trained by 250 data points from set A. The training points are inclusive of the three stages of protocol (initial rest, walking, and final rest) and are picked in the ratio of time intervals used for the respective protocol periods. Hence, the first 70 points from initial rest, first 70 points from walking, and first 110 points from the final rest were taken for training. The predictive capability of the model is gauged by computing the training error, test error, and the normalized log likelihood by testing with 100 data points (not seen by the model during training) from set $\mathrm{A}$ which are again drawn in the ratio of time intervals used for the respective protocol periods. This process is continued by increasing the number of training data points from set A. The normalized log likelihood for each set of test data is also given, calculated by dividing the value of marginal likelihood by the number of points in the test set. It can be seen that as the number of training points increase, the error value decreases and the likelihood of the data increases. This implies that with greater training points, the new model either predicts data closely or has higher confidence due to a higher density of training points.

When the model is trained on all the values of set $\mathrm{A}$ and tested with 100 points (randomly picked from set A), the training error does decrease while the likelihood also decreases slightly. This might be an indication that too much of training to the model might lead to over fitting the data. Next the model trained on the entire set A was tested on 100 points in set B which were unseen by the model. The results indicated that the test error has a substantial increase, but the likelihood function is still consistent with the uncertainty predictions of the Gaussian model. This may be because the dataset B is similar to dataset A, leading to points closer to the smooth relationship predicted by

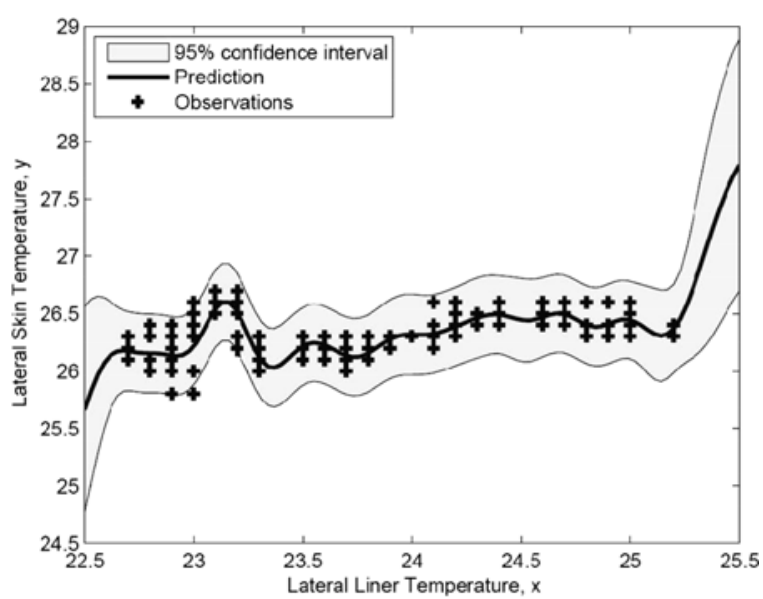

(a)

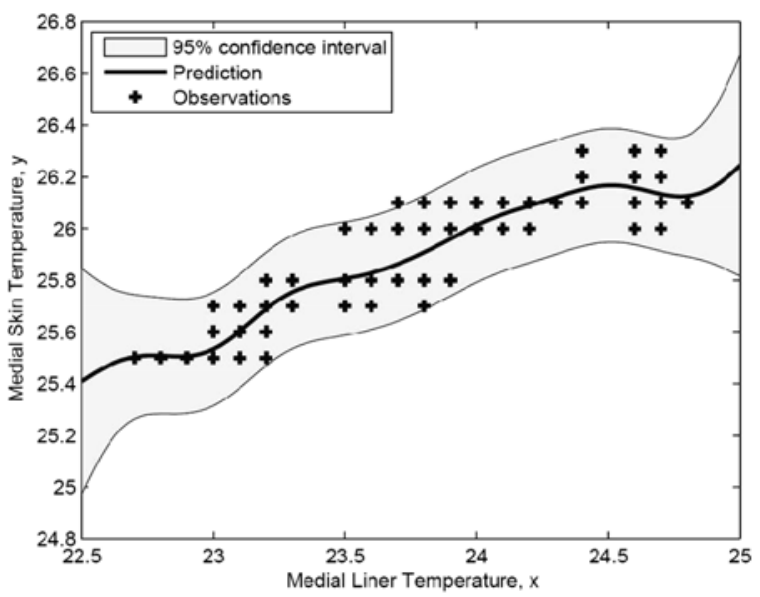

(b)

Fig. 6. Illustration of prediction with Gaussian process regression for ambient temperature of $10^{\circ} \mathrm{C}$ at (a) lateral side and (b) medial side. The test data points are given by crosses. The shaded area represents the pointwise $95 \%$ confidence region of the predictive distribution.

the Gaussian process. It was then deduced that when the model is trained on whole dataset A and hyperparameters optimized in (9), then its predictions for set B lie in the $95 \%$ confidence interval ( \pm 2 standard deviations). This is indicated in Figs. 6 and 7.

After hyperparameter optimization, the covariance hyperparameters for the lateral side at $10{ }^{\circ} \mathrm{C}$ were $[-1.27,2.92,-1.41]$ and the likelihood hyperparameter was -1.79 . The final negative log marginal likelihood (optimized) was 285.84. Table II presents the hyperparameters for the predictive model at different ambient temperatures.

A similar process was done for the medial side of residual limb skin. After the predictive algorithm was formulated, the actual and predicted data are then averaged after every five samples to create a single mean, and done till the end of all data points. This helps in smoothening out short-term fluctuations and highlighting the longer term trends.

\section{DISCUSSION}

From the raw data, it can be noted that for the ambient temperature of $10^{\circ} \mathrm{C}$, the lateral skin temperature was almost same 


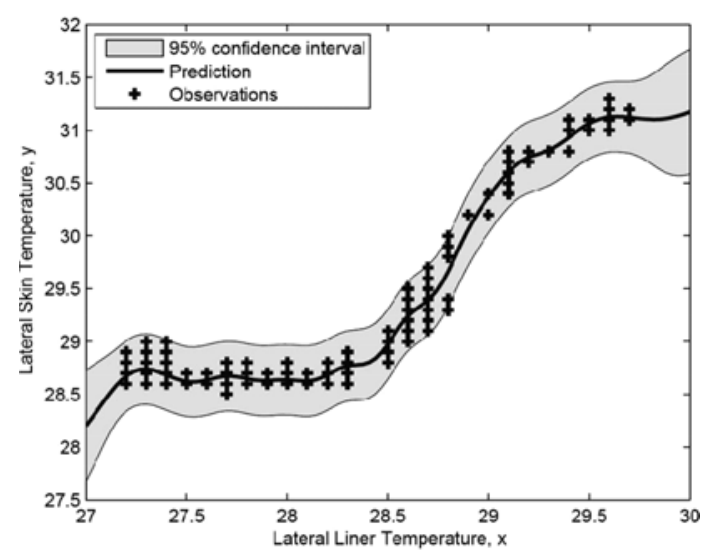

(a)

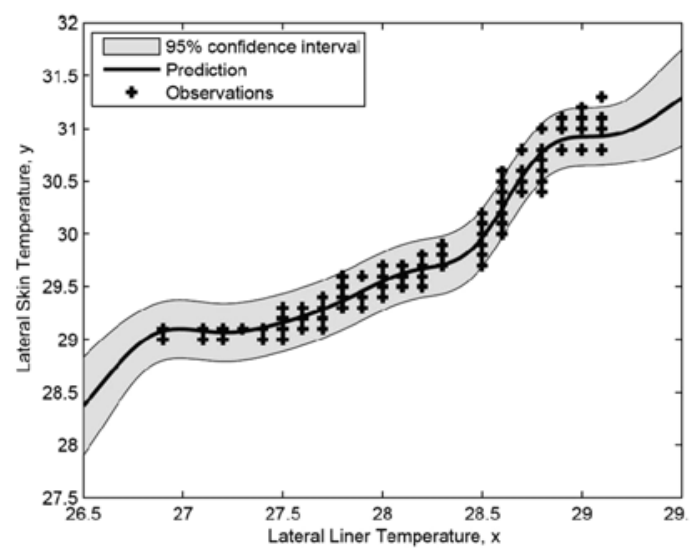

(b)

Fig. 7. Illustration of prediction with Gaussian process regression for ambient temperature of $25^{\circ} \mathrm{C}$ at (a) lateral side and (b) medial side. The test data points are given by crosses. The shaded area represents the pointwise $95 \%$ confidence region of the predictive distribution.

TABLE II

Summary of Hyperparameters For Predictive Gaussian Model

\begin{tabular}{lccccc}
\hline \hline Scenario & $\begin{array}{c}\text { Optimized } \\
\text { hyperparameters }\end{array}$ & $\begin{array}{c}\text { Initial } \\
\text { nlml }\end{array}$ & $\begin{array}{c}\text { Final } \\
\text { nlml }\end{array}$ & $\begin{array}{c}\text { Initial } \\
\text { likelihood }\end{array}$ & $\begin{array}{c}\text { Final } \\
\text { likelihood }\end{array}$ \\
\hline $\begin{array}{l}\text { Lateral } \\
\text { side at }\end{array}$ & {$[-1.27,2.92,-1.41]$} & 786.11 & 285.84 & -1.78 & -1.79 \\
$\begin{array}{l}10^{\circ} \mathrm{C} \\
\text { Medial }\end{array}$ & {$[-1.20,-0.86,1.29]$} & 747.63 & 352.61 & -2.17 & -2.21 \\
side at & & & & & \\
$10^{\circ} \mathrm{C}$ & {$[-1.86,-1.37,-1.04]$} & 538.43 & 323.78 & -1.78 & -1.80 \\
$\begin{array}{l}\text { Lateral } \\
\text { side at }\end{array}$ & & & & & \\
$25^{\circ} \mathrm{C}$ & {$[-1.35,-1.57,1.06]$} & 520.86 & 109.68 & -1.96 & -1.99 \\
$\begin{array}{l}\text { Medial } \\
\text { side at } \\
25^{\circ} \mathrm{C}\end{array}$ & & & & & \\
\hline \hline
\end{tabular}

at the start and end of the experiment. However, the medial residual limb skin temperature followed a steady profile, but the temperature at the end of the experiment was $1^{\circ} \mathrm{C}$ lower than the start. At ambient temperature of $25^{\circ} \mathrm{C}$, both the lateral and medial residual limb skin temperatures showed a steady increase in the temperature throughout the experiment. After the end of the experiment, the temperatures in both lateral and medial sides were $2.1^{\circ} \mathrm{C}$ higher than the starting. It can be seen from Figs. 2 and 5 that since the volume of the data set is big, the overall

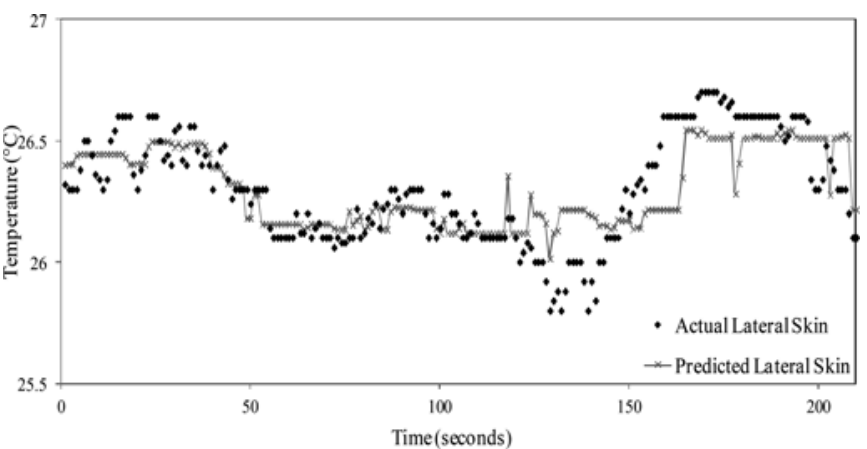

(a)

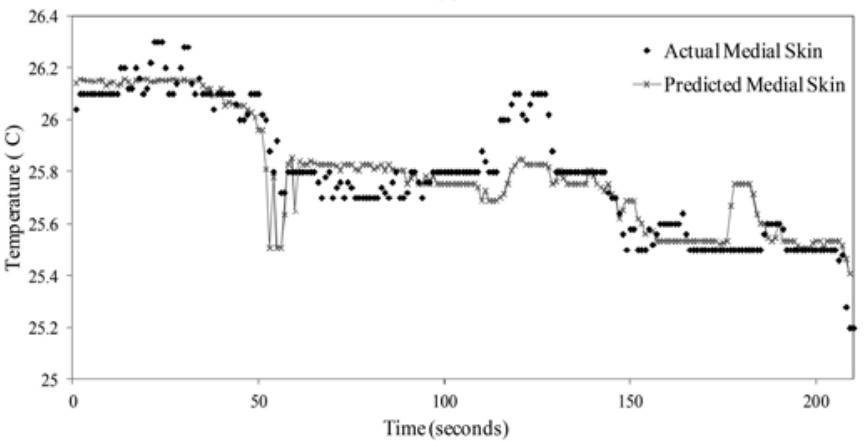

(b)

Fig. 8. Predicted residual limb skin temperature from the time averaged Gaussian process model is shown along with the actual skin temperature at lateral and medial sides in (a) and (b), respectively, at ambient temperature of $10^{\circ} \mathrm{C}$.

trend of the temperature profile is difficult to gauge. Time averaging of $5 \mathrm{~s}$ done on the recorded data helps in identifying the trend better and improves the joint probability function with an enhanced fit of the Gaussian to the data; so that more points are more accurately predicted. The actual skin temperature obtained by the Gaussian predictive model is shown in Figs. 8 and 9 for two very different ambient temperatures of $10^{\circ} \mathrm{C}$ and $25^{\circ} \mathrm{C}$, respectively.

For both these experiments done at two different ambient temperatures, our predictive model using Gaussian process provides a simple, effective, practical, and probabilistic approach to determine the unknown skin temperature of the subject within the prosthetic device from the actual liner measurements. The predictive model we developed leads to results which are in $95 \%$ confidence interval which translates to an accuracy of $\pm 0.8{ }^{\circ} \mathrm{C}$. However, this approach has certain limitations as well. Although this study was conducted on one amputee subject over a number of times to verify the influence of ambient temperatures on the in-socket temperatures, there is a need to extend it on a greater population in order to define a generic behavior. Since the residual limb temperature profile varies with changes in environmental temperatures, the Gaussian model has to be trained with individual datasets corresponding to changes in the ambient temperatures.

\section{CONCLUSION}

The challenge of noninvasively monitoring the residual limb skin temperature has been addressed in this study. This study which was conducted on a subject performing various tasks in an environmental chamber at different ambient temperatures 


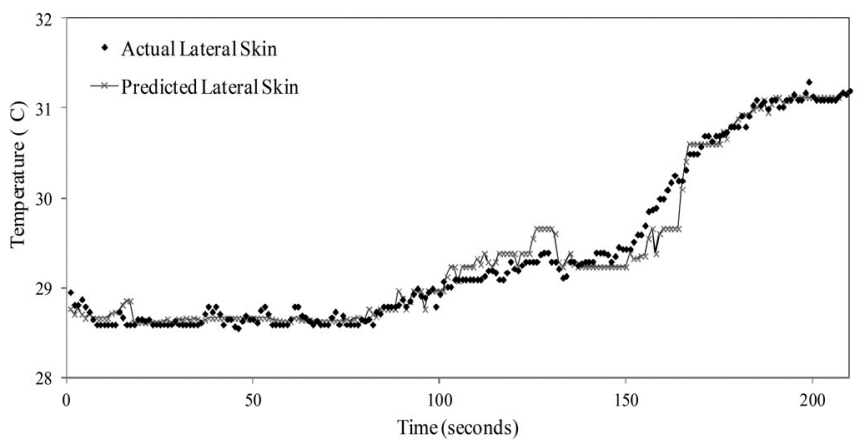

(a)

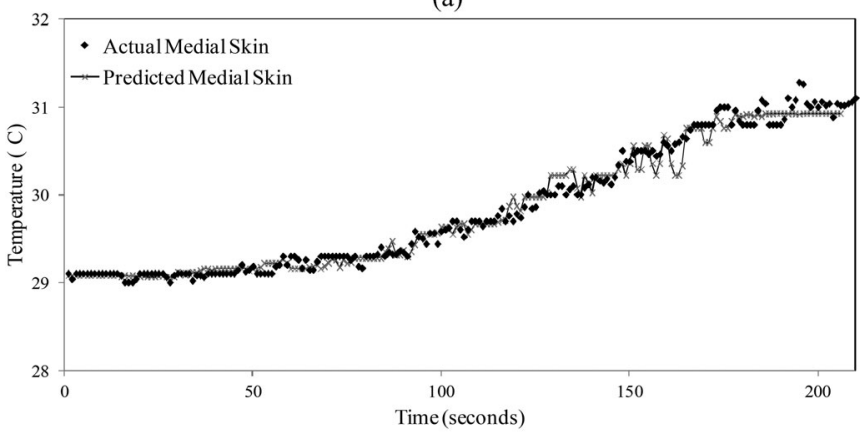

(b)

Fig. 9. Predicted residual limb skin temperature from the time averaged Gaussian process model is shown along with the actual skin temperature at lateral and medial sides in (a) and (b), respectively, at ambient temperature of $25^{\circ} \mathrm{C}$.

clearly indicated that the residual limb skin temperature and the liner temperature are majorly affected by both the ambient temperature and the activity level of the subject. The obtained data from the experimentation at ambient temperatures of $10^{\circ} \mathrm{C}$ and $25^{\circ} \mathrm{C}$ were then used to develop a predictive model using GPML. GPML is a nonparametric approach, which harnesses the training data provided to determine the underlying function. The Gaussian model was individually trained for each of the ambient temperatures on which the tests were done. With $5 \mathrm{~s}$ time averaging in order to suppress random fluctuations, the developed model provides results which lie in the 95\% confidence interval when predicting subject skin temperature within the prosthesis from the liner temperature measurements. Future scope of the work includes comparing the prediction accuracy of the Gaussian Process model with other mathematical models like the adaptive network-based fuzzy inference system using support vector regression.

\section{REFERENCES}

[1] K. Hagberg and R. Branemark, "Consequences of non-vascular transfemoral amputation: a survey of quality of life, prosthetic use and problems," Prosthetics Orthotics Int., vol. 25, no. 3, pp. 186-194, Dec. 2001.

[2] F. T. Hoaglund, H. E. Jergesen, L. Wilson, L. W. Lamoreux, and R. Roberts, "Evaluation of problems and needs of veteran lower-limb amputees in the San Francisco Bay Area during the period 1977-1980," J. Rehabil. R\&D / Veterans Admin., Dept. Med. Surgery, Rehabil. R\&D Service, vol. 20, no. 1, pp. 57-71, Jul. 1983.

[3] S. Derler and L. Gerhardt, "Tribology of skin: Review and analysis of experimental results for the friction coefficient of human skin," Tribol. Lett., vol. 45, no. 1, pp. 1-27, Jan. 2012.

[4] J. E. Sanders, B. S. Goldstein, and D. F. Leotta, "Skin response to mechanical stress adaptation rather than breakdown-A review of the literature," J. Rehabil. Res. Devel., vol. 32, no. 3, pp. 214-226, Oct. 1995.
[5] A. F. T. Mak, M. Zhang, and D. A. Boone, "State-of-the-art research in lower-limb prosthetic biomechanics-socket interface: A review," J. Rehabil. Res. Devel., vol. 38, no. 2, pp. 161-173, Mar./Apr. 2001.

[6] S. Levy, "Skin problems of the leg amputee," J. Prosthetics Orthotics, vol. 4, no. 1, pp. 37-44, Apr. 1980.

[7] R. Barnes, "Thermography," Ann. New York Acad. Sci., vol. 121, pp. 34-48, 1964.

[8] E. A. Huff, W. R. Ledoux, J. S. Berge, and J. K. Klute, "Measuring residual limb skin temperatures at the skin-prosthesis interface," J. Prosthetics Orthotics, vol. 20, no. 4, pp. 170-173, Oct. 2008.

[9] J. T. Peery, W. R. Ledoux, and G. K. Klute, "Residual-limb skin temperature in transtibial sockets," J. Rehabil. Res. Develop., vol. 42, no. 2 , pp. 147-154, Mar./Apr. 2005.

[10] G. Klute, E. Huff, and W. Ledoux, "In-socket skin temperature and perception of comfort over a whole day," presented at the Amer. Acad. Orthotists Prosthetists Annu. Meeting Sci. Symp., Seattle, WA, USA, 2007.

[11] G. Klute, J. Berge, E. Huff, and W. Ledoux, "The effect of rest and exercise on residual limb temperature," presented at the Amer. Academy Orthotists Prosthetists Annu. Meeting Sci. Symp., Chicago, IL, USA, Mar. $1-9,2006$.

[12] K. S. Maluf, R. Morley, Jr., E. Richter, J. Klaesner, and M. Mueller, "Monitoring in-shoe plantar pressures, temperature, and humidity: Reliability and validity of measures from a portable device," Archives Phys. Med. Rehabil., vol. 82, no. 8, pp. 1119-1129, Aug. 2001.

[13] R. Emrich and K. Slater, "Comparative analysis of below-knee prosthetic socket liner materials," J. Med. Eng. Technol., vol. 22, no. 2, pp. 94-98, Jan. 1998.

[14] M. Gerschutz, M. Haynes, D. Nixon, and J. Colvin, "Strength evaluation of prosthetic check sockets, copolymer sockets, and definitive laminated sockets," J. Rehabil. Res. Develop., vol. 49, no. 3, pp. 405-426, May 2012.

[15] M. Gerschutz, M. Haynes, D. Nixon, and J. Colvin, "Tensile strength and impact resistance properties of materials used in prosthetic check sockets, copolymer sockets, and definitive laminated sockets," J. Rehabil. Res. Develop., vol. 48, no. 8, pp. 987-1004, Nov. 2011.

[16] D. Taylor, "Lower limb prosthetics-Mechanical testing prosthetic sockets," presented at the Acad. Orthotist Prosthetists Annu. Meeting Sci. Symp., Orlando, FL, USA, Mar. 16-19, 2005.

[17] G. K. Klute, G. I. Rowe, A. V. Mamishev, and W. R. Ledoux, "The thermal conductivity of prosthetic sockets and liners," Prosthetics Orthotics Int., vol. 31, no. 3, pp. 292-299, Sep. 2007.

[18] A. Shitzer and R. Eberhart, Heat Transfer in Medicine and Biology: Analysis and Applications. New York, NY, USA: Plenum Press, 1983.

[19] Y. Houdas and E. Ring, Human Body Temperature Its Measurement and Regulation. New York, NY, USA: Plenum Press, 1982.

[20] J. Peery, G. Klute, J. Blevins, and W. Ledoux, "A three-dimensional finite element model of the transtibial residual limb and prosthetic socket to predict skin temperatures," IEEE Trans. Neural Syst. Rehabil. Eng., vol. 14, no. 3, pp. 336-343, Sep. 2006.

[21] C. E. Rasmussen and C. K. I. Williams. (2013, Jan.). Gaussian Processes for Machine Learning software. [Online]. Available: www.gaussianprocess.org/gpml/

[22] C. K. I. Williams and C. E. Rasmussen, "Gaussian processes for regression," in Neural Information Processing Systems, vol. 8, Cambridge, MA, USA, MIT Press, 1996, pp. 514-520.

[23] C. E. Rasmussen and C. K. I. Williams, Gaussian Processes For Machine Learning. Adaptive Computation and Machine Learning. Cambridge, MA, USA: MIT Press, 2005.

[24] M. N. Gibbs, "Bayesian Gaussian processes for regression and classification," Ph.D. dissertation, Dept. of Physics, Univ. Cambridge, Cambridge, U.K., 1997.

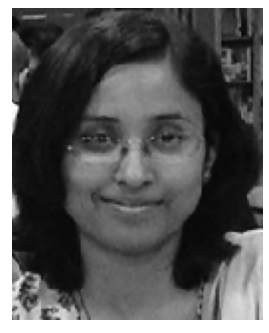

Neha Mathur ( $\mathrm{S}^{\prime} 03$ ) received the B.E. degree in electrical engineering from Bharati Vidyapeeth University, Pune, India, in 2005, and the M.S. degree from the University of New Hampshire, Durham, NH, USA, in 2008. She is currently working toward the $\mathrm{Ph} . \mathrm{D}$. degree in electronic and electrical engineering at University of Strathclyde, Glasgow, U.K.

From 2008 to 2011, she joined Corus Steel, Port Talbot, U.K., where she was a Process Control and Automation Engineer. Her research interest includes development of sensor systems for data monitoring, analysis, and prediction of residual limb health in prosthetics. 


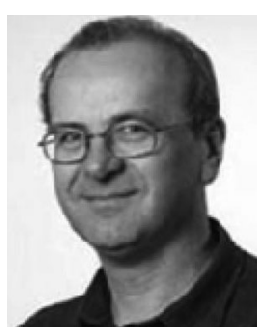

Ivan Glesk (M'01-SM'01) received the Ph.D. degree in quantum electronics and optics from Comenius University, Bratislava, Slovakia, in 1989, and the D.Sc. degree from the Slovak Academy of Sciences, Bratislava.

In 1986, he joined the Department of Experimental Physics, Comenius University, where he later became a Professor of physics, and was involved in the research in areas of nonlinear optics, laser physics, and using LIDAR methods for remote sensing in the atmosphere. In 1991, he joined Princeton University, Princeton, NJ, USA, where he was a Manager of the Lightwave Communication Research Laboratory and Senior Research Scholar in the Department of Electrical Engineering. In 2007, he joined University of Strathclyde, Glasgow, U.K., as a Professor of Broadband Communication Systems in the Department of Electronic and Electrical Engineering. He is author and co-author of more than 290 publications, 16 book chapters and holds five patents. His current research interests include biophotonics, sensors, applications of ultrashort pulses in data communication, ultrafast optical signal processing, and optical interconnects.

Dr. Glesk is on the Editorial Board of the International Journal of Optics and Optica Aplicata. He is a recipient of the International Research and Exchanges Board (IREX) Fellowship.

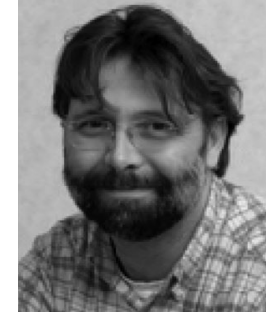

Arjan Buis graduated and started his Ph.D. research at the University of Strathclyde, Glasgow, U.K., in 1997.

He is a Senior Research Fellow at the Department of Biomedical Engineering, University of Strathclyde. His current research relates to improving our understanding of the biomechanical mechanisms that contribute to the generation and control of load transfer forces dealing with the subject "where man meets machine" and especially the area of prosthetic socket fit. Examples of this are evident in his research related to the capture of proof of the dynamic mechanical environment at the stump-socket interface, within the deeper structures of the stump and the overall impact of this approach on amputee gait performance and acceptance. Besides the biomechanical interests, he is also developing a portfolio in relation to component design related topics and includes; "Smart" materials applicable in rehabilitation technology and development of specific prosthetic components using alternative materials and composites. In addition, he is leading a research group dealing with integrated intelligent sensor systems to enable built-in data monitoring of prosthetics and orthotics as a means to maintain optimum performance and quality of life. 